\title{
CIÊNCIANATURA
}

\section{A distribuição Lindley potência inversa: diferentes métodos de estimação}

\author{
The power inverse Lindley distribution: different methods of estimation
}

\author{
André F. B. Menezes ${ }^{* 1}$, Josmar Mazucheli ${ }^{1}$ e Kelly V. P. Barco ${ }^{2}$ \\ ${ }^{1}$ Department of Statistics, Maringá State University, Maringá, P.R., Brazil \\ ${ }^{2}$ Union Facult of Campo Mourão, Campo Mourão, P.R., Brazil
}

\section{Resumo}

Nos últimos anos diversas distribuições de probabilidade foram propostas na literatura com propósitos de se obter funções densidade e de risco mais flexíveis. Por exemplo, Ghitany et al. (2013) propuseram uma generalização da distribuição Lindley e a nomearam de distribuição Lindley potência enquanto que Sharma et al. (2015a) propuseram a distribuição Lindley inversa. A partir destas duas generalizações, Barco et al. (2017) estudaram a distribuição Lindley potência inversa, também chamada por Sharma et al. (2015b) de Lindley inversa generalizada. Neste artigo, considerando a proposta de Barco et al. (2017), avaliou-se, via simulações Monte Carlo, o viés e acurácia de nove métodos de estimação (o método da máxima verossimilhança e oito outros baseados nas distâncias entre as funções de distribuições empíricas e teóricas). Os resultados provenientes do estudo de simulação indicam melhor desempenho do método de estimação baseado na estatística do teste de Anderson-Darling. Esta conclusão também é observada na análise de dois conjuntos de dados reais.

Palavras-chave: Distribuição Lindley potência inversa, Métodos de estimação, Verossimilhança, Simulação Monte Carlo.

\section{Abstract}

In the last years several probability distributions have been proposed in the literature, especially with the aim of obtaining models that are more flexible relative to the behaviors of the density and hazard rate functions. For instance, Ghitany et al. (2013) proposed a new generalization of the Lindley distribution, called power Lindley distribution, whereas Sharma et al. (2015a) proposed the inverse Lindley distribution. From these two generalizations Barco et al. (2017) studied the inverse power Lindley distribution, also called by Sharma et al. (2015b) as generalized inverse Lindley distribution. Considering the inverse power Lindley distribution, in this paper is evaluate the performance, through Monte Carlo simulations, with respect to the bias and consistency of nine different methods of estimations (the maximum likelihood method and eight others based on the distance between the empirical and theoretical cumulative distribution function). The numerical results showed a better performance of the estimation method based on the Anderson-Darling test statistic. This conclusion is also observed in the analysis of two real data sets.

Keywords: Power inverse Lindley distribution, Methods of estimation, Likelihood, Monte Carlo simulation. 


\section{Introdução}

A distribuição Lindley proposta por Lindley (1958) pode ser obtida pela mistura entre a distribuição exponencial, com parâmetro de escala $\beta$, e a distribuição gama, com parâmetro de forma igual a 2 e de escala $\beta$. Segundo esta formulação a função densidade de probabilidade é escrita na forma

$$
f(x \mid \beta)=p f_{1}(x \mid \beta)+(1-p) f_{2}(x \mid 2, \beta)
$$

$\operatorname{com} p=\frac{\beta}{1+\beta}, f_{1}(x \mid \beta)=\beta e^{-\beta x}$ e $f_{2}(x \mid 2, \beta)=\beta^{2} x e^{-\beta x}$. A partir destes termos tem-se

$$
f(x \mid \beta)=\frac{\beta^{2}}{1+\beta}(1+x) e^{-\beta x} .
$$

De (1), a função de distribuição acumulada e de risco podem ser escritas, respectivamente, como

$$
F(x \mid \beta)=1-\left(1+\frac{\beta x}{1+\beta}\right) e^{-\beta x}
$$

$\mathrm{e}$

$$
h(x \mid \beta)=\frac{\beta^{2}(1+x)}{\beta(1+x)+1} .
$$

Desde sua reintrodução na literatura estatística por Ghitany et al. (2008), a distribuição Lindley vem sendo intensivamente generalizada por vários autores. Por exemplo, a distribuição Lindley inversa, proposta por Sharma et al. (2015a), é obtida a partir do inverso de uma variável aleatória com distribuição Lindley. Matematicamente, se uma variável aleatória $Y$ tem distribuição Lindley então a variável aleatória $X=Y^{-1}$ tem distribuição Lindley inversa com funções densidade, de distribuição acumulada e de risco definidas, respectivamente, por

$$
\begin{aligned}
& f(x \mid \beta)=\frac{\beta^{2}}{1+\beta}\left(\frac{1+x}{x^{3}}\right) e^{-\frac{\beta}{x}}, \\
& F(x \mid \beta)=\left(1+\frac{\beta}{1+\beta} \frac{1}{x}\right) e^{-\frac{\beta}{x}}
\end{aligned}
$$

e

$$
h(x \mid \beta)=\frac{\beta^{2}(1+x)}{x^{2}\left[-\beta+x(1+\beta)\left(e^{\beta / x}-1\right)\right]} .
$$

Outra generalização, proposta por Ghitany et al. (2013) e nomeada de distribuição Lindley potência, considera a potência de uma variável aleatória com distribuição Lindley, ou seja $X=Y^{\frac{1}{\alpha}}$ em que $\alpha>0$. Do mesmo modo que a distribuição Lindley, a distribuição Lindley potência pode ser obtida pela mistura de duas distribuições, sendo elas, a distribuição Weibull com parâmetro de forma $\alpha$ e de escala $\beta$ e a distribuição gama generalizada com parâmetros de forma 2 e $\alpha$ e de escala $\beta$. Segundo esta formulação os termos de (1) são, respectivamente, $p=\frac{\beta}{1+\beta}, f_{1}(x \mid \alpha, \beta)=\alpha \beta x^{\alpha-1} e^{-\beta x^{\alpha}}$ e $f_{2}(x \mid 2, \alpha, \beta)=\alpha \beta^{2} x^{2 \alpha-1} e^{-\beta x^{\alpha}}$. Explicitamente, as funções densidade, de distribuição acumulada e de risco são escritas, respectivamente, como

$$
\begin{aligned}
& f(x \mid \alpha, \beta)=\frac{\alpha \beta^{2}}{1+\beta}\left(1+x^{\alpha}\right) x^{\alpha-1} e^{-\beta x^{\alpha}}, \\
& F(x \mid \alpha, \beta)=1-\left(1+\frac{\beta x^{\alpha}}{1+\beta}\right) e^{-\beta x^{\alpha}}
\end{aligned}
$$

$\mathrm{e}$

$$
h(x \mid \alpha, \beta)=\alpha \beta^{2} \frac{\left(1+x^{\alpha}\right) x^{\alpha-1}}{\beta\left(1+x^{\alpha}\right)+1} .
$$

A partir da distribuição Lindley inversa, Barco et al. (2017) formulou a distribuição Lindley potência inversa aplicando a transformação $X=Y^{\frac{1}{\alpha}}$ em que $Y$ tem distribuição Lindley inversa e $\alpha>0$. A função densidade de probabilidade da distribuição Lindley potência inversa pode ser escrita na forma

$$
f(x \mid \alpha, \beta)=\frac{\alpha \beta^{2}}{1+\beta}\left(\frac{1+x^{\alpha}}{x^{2 \alpha+1}}\right) e^{-\frac{\beta}{x^{\alpha}}} .
$$

A função de distribuição acumulada e de risco são escritas, respectivamente, como

$$
F(x \mid \alpha, \beta)=\left(1+\frac{\beta}{1+\beta} \frac{1}{x^{\alpha}}\right) e^{-\frac{\beta}{x^{\alpha}}}
$$




$$
h(x \mid \alpha, \beta)=\frac{\alpha \beta^{2}\left(1+x^{-\alpha}\right)}{x\left[-\beta+x^{\alpha}(1+\beta)\left(e^{\frac{\beta}{x^{\alpha}}}-1\right)\right]} .
$$

Os comportamentos de (2) e (4), considerando alguns valores para $\alpha$ e $\beta$, são apresentados nas Figuras 1 e 2 , respectivamente. É fácil notar a importância da adição do parâmetro $\alpha$ nas funções de densidade e de risco da distribuição Lindley inversa. É importante mencionar que a unimodalidade de (2) e (4), para todo $\alpha>0$ e $\beta>0$, foram matematicamente verificadas em Barco et al. (2017).
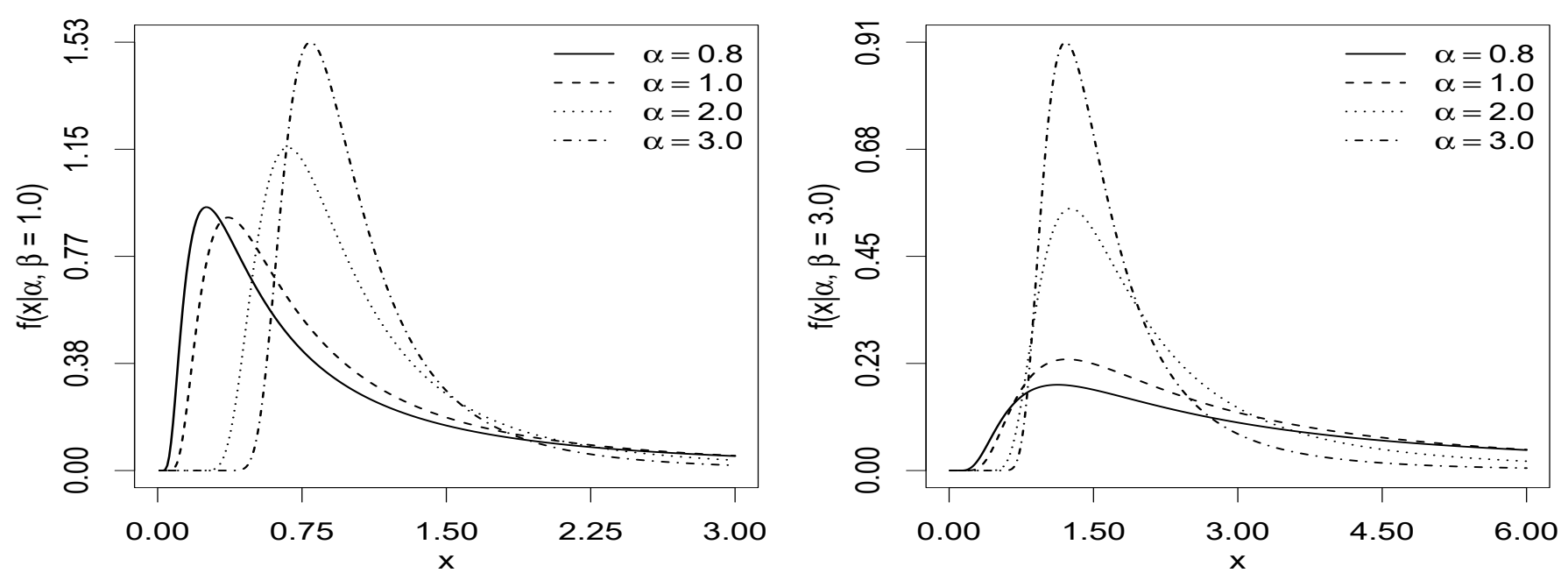

Figure 1: Comportamento da função densidade de probabilidade da distribuição Lindley potência inversa.
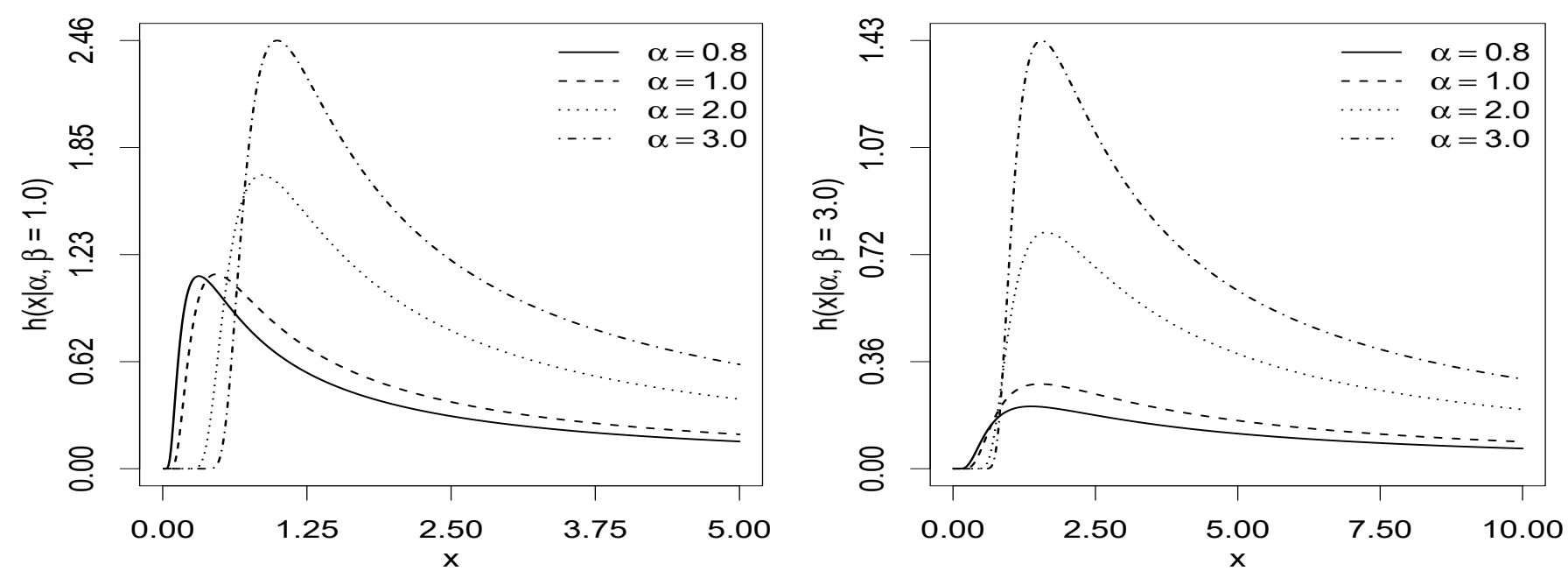

Figure 2: Comportamento da função de risco da distribuição Lindley potência inversa.

Sob o ponto de vista da inferência paramétrica, a estimação dos parâmetros de uma distribuição de probabilidade é o ponto de partida em qualquer análise de dados. Dentre os diversos métodos de estimação, seguramente, o método da máxima verossimilhança é o mais utilizado (ver, por exemplo, Lehmann e Casella, 1998; Pawitan, 2001; Rohde, 2014). O método da máxima verossimilhança foi o utilizado por Barco et al. (2017) para estimar os parâmetros $\alpha$ e $\beta$ da distribuição Lindley potência inversa. Ainda nesse trabalho foram estimados o viés e a acurácia de $\widehat{\alpha}$ e $\widehat{\beta}$. É importante mencionar que vários outros métodos de estimação de parâmetros estão disponíveis na literatura (ver, por exemplo, Gupta e Kundu, 2001; Mazucheli et al., 2017) e nem sempre é fácil identificar o "melhor" em termos, por exemplo, de menor viés e maior acurácia.

Neste artigo, considerando a distribuição Lindley potência inversa, são estimados, via simulações Monte Carlo, o viés e o erro quadrático médio dos estimadores de $\alpha$ e $\beta$, obtidos pelo método da máxima verossimilhança e oito outros baseados nas distâncias entre as funções de distribuição empírica e teórica. A originalidade deste trabalho deve-se ao fato de que, em princípio, nenhum trabalho anterior comparou estes métodos na estimação dos parâmetros da distribuição Lindley potência inversa. 
A prática de comparar diferentes métodos de estimação vem sendo intensivamente explorada na literatura, por exemplo, sem exaustão, em Gupta e Kundu (2001), Kundu e Raqab (2005) e Teimouri et al. (2013) foram considerados cinco métodos e as distribuições exponencial generalizada, Rayleigh generalizada e Weibull respectivamente. Em Mazucheli et al. (2013) foram considerados quatro métodos e a distribuição Lindley ponderada, do Espirito-Santo e Mazucheli (2015) consideraram seis métodos e a distribuição Lindley Marshall-Olkin e em Mazucheli et al. (2017) e Dey et al. (2017) foram considerados dez métodos e as distribuições exponencial Marshall-Olkin e Kumaraswamy, respectivamente.

\section{Métodos de estimação}

Nesta seção são descritos sucintamente os nove métodos utilizados neste artigo para para estimar os parâmetros $\alpha$ e $\beta$ da distribuição Lindley potência inversa. Para todos os métodos foram considerados que ambos os parâmetros são desconhecidos.

\subsection{Método da máxima verossimilhança}

Sejam $\boldsymbol{x}=\left(x_{1}, \ldots, x_{n}\right)$ os valores observados de uma variável aleatória com distribuição de probabilidade Lindley potência inversa. A função log-verossimilhança pode ser escrita na forma

$$
l(\alpha, \beta \mid \boldsymbol{x})=n[\log \alpha+2 \log \beta-\log (1+\beta)]+\sum_{i=1}^{n} \log \left(1+x_{i}^{\alpha}\right)-(2 \alpha+1) \sum_{i=1}^{n} \log x_{i}-\beta \sum_{i=1}^{n} x_{i}^{-\alpha} .
$$

As estimativas de máxima verossimilhança $\widehat{\alpha}_{E M V}$ e $\widehat{\beta}_{E M V}$ de $\alpha$ e $\beta$, respectivamente, maximizam (5) e são as raízes do sistema de equações

$$
\left\{\begin{aligned}
\frac{\partial}{\partial \alpha} l(\alpha, \beta \mid \boldsymbol{x}) & =\frac{n}{\alpha}+\sum_{i=1}^{n} \frac{x_{i}^{\alpha} \log x_{i}}{1+x_{i}^{\alpha}}-2 \sum_{i=1}^{n} \log x_{i}+\beta \sum_{i=1}^{n} x_{i}^{-\alpha} \log x_{i}=0 \\
\frac{\partial}{\partial \beta} l(\alpha, \beta \mid \boldsymbol{x}) & =\frac{n(2+\beta)}{\beta(1+\beta)}-\sum_{i=1}^{n} x_{i}^{-\alpha}=0 .
\end{aligned}\right.
$$

A partir de $\frac{\partial}{\partial \beta} l(\alpha, \beta \mid \boldsymbol{x})$ tem-se

$$
\left(\sum_{i=1}^{n} x_{i}^{-\alpha}\right) \beta^{2}+\left(\sum_{i=1}^{n} x_{i}^{-\alpha}-n\right) \beta-2 n=0 .
$$

Consequentemente a estimativa de máxima verossimilhança de $\beta, \widehat{\beta}_{E M V}$, provém da equação

$$
\widehat{\beta}\left(\widehat{\alpha}_{E M V}\right)=\frac{-\left(\sum_{i=1}^{n} x_{i}^{-\widehat{\alpha}_{E M V}}-n\right)+\sqrt{\left(\sum_{i=1}^{n} x_{i}^{-\widehat{\alpha}_{E M V}}-n\right)^{2}+8 n \sum_{i=1}^{n} x_{i}^{-\widehat{\alpha}_{E M V}}}}{2 \sum_{i=1}^{n} x_{i}^{-\widehat{\alpha}_{E M V}}},
$$

enquanto que $\widehat{\alpha}_{E M V}$ é raiz da equação

$$
\frac{n}{\alpha}+\sum_{i=1}^{n} \frac{x_{i}^{\alpha} \log x_{i}}{1+x_{i}^{\alpha}}-2 \sum_{i=1}^{n} \log x_{i}+\widehat{\beta}\left(\alpha_{E M V}\right) \sum_{i=1}^{n} x_{i}^{-\alpha} \log x_{i}
$$

Os intervalos de confiança $100 \times(1-\delta) \%, 0<\delta<1$, para $\alpha$ e $\beta$, baseados na normalidade assintótica dos estimadores de máxima verossimilhança são obtidos, respectivamente, por

$$
\widehat{\alpha} \pm z_{\delta / 2} \sqrt{\widehat{\operatorname{Var}}(\widehat{\alpha})} \quad \text { e } \quad \widehat{\beta} \pm z_{\delta / 2} \sqrt{\widehat{\operatorname{Var}}(\widehat{\beta})}
$$

em que $\operatorname{Var}(\cdot)$ denotam os elementos da diagonal principal, da matriz inversa informação de Fisher, $\boldsymbol{I}^{-1}(\alpha, \beta)$ estimada.

Conforme apresentado por Barco et al. (2017), os elementos da matriz de informação de Fisher, $\boldsymbol{I}(\alpha, \beta)$, da distribuição Lindley potência inversa são

$$
\boldsymbol{I}_{11}=\frac{1}{\alpha^{2}}+\frac{1}{\alpha^{2}(1+\beta)}\left\{\beta\left[(\psi(2)-\log \beta)^{2}+\zeta(2,2)\right]+2\left[(\psi(3)-\log \beta)^{2}+\zeta(2,3)\right]-\beta\left[(\psi(1)-\log \beta)^{2}+\zeta(2,1)\right]+\beta^{2} J(\beta)\right\}
$$




$$
\begin{aligned}
& \boldsymbol{I}_{22}=\frac{\beta^{2}+4 \beta+2}{\beta^{2}(1+\beta)^{2}}, \\
& \boldsymbol{I}_{12}=-\frac{\beta[\psi(2)-\log \beta]+2[\psi(3)-\log \beta]}{\alpha \beta(1+\beta)} .
\end{aligned}
$$

em que $\psi(\cdot)$ é a função digama e $\zeta(\cdot)$ é a função zeta de Riemann.

\subsection{Métodos das distâncias mínimas}

Os métodos alternativos ao da máxima verossimilhança discutidos nesta subseção utilizam as estatísticas dos testes de Cramér-von Mises (CvM), Kolmogorov-Smirnov (KS), Anderson-Darling (AD) e cinco variações da estatística do teste de Anderson-Darling cujos acrônimos são ADL, AD2R, AD2L e AD2. A idéia nestes métodos é determinar os valores dos parâmetros que minimizam a distância entre expressões envolvendo a função de distribuição teórica $F\left(x_{i} \mid \alpha, \beta\right)$ e a empírica (ver, por exemplo, D’Agostino e Stephens (1986) e Lucenõ (2006) para maiores detalhes). As expressões para cada um destes métodos estão apresentadas na Tabela 1

Table 1: Expressões dos métodos de estimação baseados nas distâncias mínimas.

\begin{tabular}{c|l}
\hline Acrônimo & \multicolumn{1}{c}{ Expressões } \\
\hline $\mathrm{CvM}$ & $W_{n}^{2}=\frac{1}{12 n}+\sum_{i=1}^{n}\left(z_{i}-\frac{2 i-1}{2 n}\right)^{2}$ \\
$\mathrm{KS}$ & $D_{n}=\frac{1}{2 n}+\max _{1 \leq i \leq n}\left|z_{i}-\frac{2 i-1}{2 n}\right|$ \\
$\mathrm{AD}$ & $A_{n}^{2}=-n-\frac{1}{n} \sum_{i=1}^{n}(2 i-1)\left[\log z_{i}+\log \left(1-z_{n+1-i}\right)\right]$ \\
$\mathrm{ADR}$ & $R^{2}=\frac{n}{2}-2 \sum_{i=1}^{n} z_{i}-\frac{1}{n} \sum_{i=1}^{n}(2 i-1) \log \left(1-z_{n+1-i}\right)$ \\
$\mathrm{ADL}$ & $L_{n}^{2}=-\frac{3 n}{2}+2 \sum_{i=1}^{n} z_{i}-\frac{1}{n} \sum_{i=1}^{n}(2 i-1) \log z_{i}$ \\
$\mathrm{AD} 2 \mathrm{R}$ & $r_{n}^{2}=2 \sum_{i=1}^{n} \log \left(1-z_{i}\right)+\frac{1}{n} \sum_{i=1}^{n} \frac{2 i-1}{1-z_{n+1-i}}$ \\
$\mathrm{AD} 2 \mathrm{~L}$ & $l_{n}^{2}=2 \sum_{i=1}^{n} \log z_{i}+\frac{1}{n} \sum_{i=1}^{n} \frac{2 i-1}{z_{i}}$ \\
$\mathrm{AD} 2$ & $a_{n}^{2}=2 \sum_{i=1}^{n}\left[\log z_{i}+\log \left(1-z_{i}\right)\right]+\frac{1}{n} \sum_{i=1}^{n}\left(\frac{2 i-1}{z_{i}}+\frac{2 i-1}{1-z_{n+1-i}}\right)$ \\
\hline
\end{tabular}

em que $x_{1}, \ldots, x_{n}$ são as observações i.i.d. da distribuição da variável aleatória $X$ com função de distribuição acumulada (3), $x_{(1)} \leq \cdots \leq x_{(n)}$ são as respectivas estatísticas de ordem da amostra e $z_{i}=F\left(x_{(i)} \mid \alpha, \beta\right)$.

A título de ilustração, a seguir são apresentadas as expressões usadas na estimação de $\alpha$ e $\beta$ a partir dos métodos de Cramér-von-Mises, Kolmogorov-Smirnov e Anderson-Darling.

- Método de Cramér-von-Mises: Sejam $x_{(1)} \leq \cdots \leq x_{(n)}$ as correspondentes estatísticas de ordem de $x_{1}, \ldots, x_{n}$. As estimativas $\widehat{\alpha}_{\mathrm{CvM}}$ e $\widehat{\beta}_{\mathrm{CvM}}$ de $\alpha$ e $\beta$, respectivamente, são os valores que minimizam a soma de quadrados das diferenças entre $F\left(x_{(i)} \mid \alpha, \beta\right)$ e a expressão de CvM, apresentada na Tabela 1. Matematicamente tem-se

$$
W_{n}^{2}(\alpha, \beta)=\frac{1}{12 n}+\sum_{i=1}^{n}\left(z_{i}-\frac{2 i-1}{2 n}\right)^{2}
$$

de forma que $\widehat{\alpha}_{\text {CvM }}$ e $\widehat{\beta}_{\text {CvM }}$ são raízes dos sistema

$$
\left\{\begin{array}{l}
\sum_{i=1}^{n}\left(z_{i}-\frac{2 i-1}{2 n}\right) \Delta_{1}\left(x_{(i)} \mid \alpha, \beta\right)=0 \\
\sum_{i=1}^{n}\left(z_{i}-\frac{2 i-1}{2 n}\right) \Delta_{2}\left(x_{(i)} \mid \alpha, \beta\right)=0
\end{array}\right.
$$

em que $\Delta_{1}\left(x_{(i)} \mid \alpha, \beta\right)$ e $\Delta_{2}\left(x_{(i)} \mid \alpha, \beta\right)$ são definidas, respectivamente, por

$$
\Delta_{1}\left(x_{(i)} \mid \alpha, \beta\right)=\frac{\beta^{2} \log \left(x_{(i)}\right) e^{-\frac{\beta}{\alpha^{2}}}}{1+\beta}\left(\frac{1}{x_{(i)}^{\alpha}}+\frac{1}{x_{(i)}^{2 \alpha}}\right) \quad \text { e } \quad \Delta_{2}\left(x_{(i)} \mid \alpha, \beta\right)=-\frac{\beta e^{-\frac{\beta}{\alpha^{2}}}}{x_{(i)}^{\alpha}(1+\beta)^{2}}\left(2+\beta+\frac{1+\beta}{x_{(i)}^{\alpha}}\right) .
$$


- Método de Kolmogorov-Smirnov: As estimativas $\widehat{\alpha}_{K S}$ e $\widehat{\beta}_{K S}$ de $\alpha$ e $\beta$, obtidas da estatística de Kolmogorov-Smirnov, minimizam a equação

$$
D_{n}(\alpha, \beta)=\frac{1}{2 n}+\max _{1 \leq i \leq n}\left(\left|z_{i}-\frac{i}{n}\right|,\left|z_{i}-\frac{i-1}{n}\right|\right) .
$$

- Método de Anderson-Darling: A partir da estatística de Anderson-Darling, as estimativas $\widehat{\alpha}_{A D}$ e $\widehat{\beta}_{A D}$ de $\alpha$ e $\beta$ minimizam a equação

$$
A_{n}^{2}(\alpha, \beta)=-n-\frac{1}{n} \sum_{i=1}^{n}(2 i-1)\left[\log z_{i}+\log \left(1-z_{n+1-i}\right)\right]
$$

obtidas a partir da resolução do sistema não-linear

$$
\left\{\begin{array}{l}
\sum_{i=1}^{n}(2 i-1)\left[\frac{\Delta_{1}\left(x_{(i)} \mid \alpha, \beta\right)}{z_{i}}-\frac{\Delta_{1}\left(x_{(n+1-i)} \mid \alpha, \beta\right)}{1-z_{n+1-i}}\right]=0 \\
\sum_{i=1}^{n}(2 i-1)\left[\frac{\Delta_{2}\left(x_{(i)} \mid \alpha, \beta\right)}{z_{i}}-\frac{\Delta_{2}\left(x_{(n+1-i)} \mid \alpha, \beta\right)}{1-z_{n+1-i}}\right]=0
\end{array}\right.
$$

em que $\Delta_{1}(\cdot \mid \alpha, \beta)$ e $\Delta_{2}(\cdot \mid \alpha, \beta)$ estão definidas em (6).

\section{Estudo de simulação}

Nesta seção são apresentados os resultados dos estudos de simulações usados para avaliar a performance dos nove métodos de estimação acima descritos. Considera-se amostras de tamanhos 20, 50, 80,110,140, 170 e 200 e alguns dos valores usados na construção das Figuras 1 e $2,(\boldsymbol{\alpha}, \boldsymbol{\beta})=(0.8,1.0),(0.8,3.0),(2.0,0.8)$ e $(2.0,3.0)$. Os resultados de outras combinações de $\alpha$ e $\beta$ foram omitidas, por motivos de espaço e por não terem gerados resultados cujas conclusões diferem das apresentadas. Para cada combinação foram geradas $B=100,000$ amostras utilizando a expressão $x_{i}=F^{-1}\left(u_{i}\right), i=1, \ldots, n$, em que:

$$
x_{i}=\left[-1-\frac{1}{\beta}-\frac{1}{\beta} W_{-1}\left(-u_{i}(1+\beta) e^{-(1+\beta)}\right)\right]^{-\frac{1}{\alpha}},
$$

$u_{i}$ são valores gerados de uma variável aleatória uniforme no intervalo $(0,1)$ e $W_{-1}$ é a função Lambert W. Vale ressaltar que (7) está disponível na biblioteca LindleyR (Mazucheli et al., 2016), do sistema R (R Core Team, 2017).

A partir das $B=100,000$ estimativas de $\alpha$ e $\beta$, obtidas por cada um dos nove métodos, foram estimados o viés e o erro quadrático médio cujas expressões são

$$
\begin{array}{rlrl}
\text { Viés }(\widehat{\alpha}) & =\frac{1}{B} \sum_{i=1}^{B}\left(\widehat{\alpha}_{i}-\alpha\right), & & \text { Viés }(\widehat{\beta})=\frac{1}{B} \sum_{i=1}^{B}\left(\widehat{\beta}_{i}-\beta\right), \\
\operatorname{EQM}(\widehat{\alpha})=\frac{1}{B} \sum_{i=1}^{B}\left(\widehat{\alpha}_{i}-\alpha\right)^{2}, & \operatorname{EQM}(\widehat{\beta})=\frac{1}{B} \sum_{i=1}^{B}\left(\widehat{\beta}_{i}-\beta\right)^{2} .
\end{array}
$$

Todo o estudo de simulação foi executado em Ox Console, versão 7.09, (Doornik, 2007), utilizando-se a função MaxBFGS. O procedimento completo usado na estimação de $\alpha$ e $\beta$ é descrito a seguir.

Considere niter $=50$ (número de iterações), error $=0.00001$ (maior erro tolerável até a convergência do método BFGS), $\boldsymbol{n}=(20,50,80,110,140,170,200), \boldsymbol{\alpha}=(0.8,1.0,2.0), \boldsymbol{\beta}=(0.8,3.0), \boldsymbol{M}=(\mathrm{EMV}, \mathrm{CvM}, \mathrm{KS}, \mathrm{AD}, \mathrm{ADR}, \mathrm{ADL}, \mathrm{AD} 2 \mathrm{R}, \mathrm{AD} 2 \mathrm{~L}$, AD2) e $B=100,000$ replicações.

$$
\begin{aligned}
& \text { for } h=1, \ldots, \text { length }(\boldsymbol{\beta}) \\
& \left\{\begin{aligned}
\beta=\boldsymbol{\beta}[h] \\
\text { for } i=1, \ldots, \text { length }(\boldsymbol{\alpha})
\end{aligned}\right. \\
& \left\{\begin{array}{l}
\alpha=\boldsymbol{\alpha}[i] \\
\text { for } j=1, \ldots, \text { length }(\boldsymbol{n}) \\
\{\quad n=\boldsymbol{n}[j]
\end{array}\right.
\end{aligned}
$$




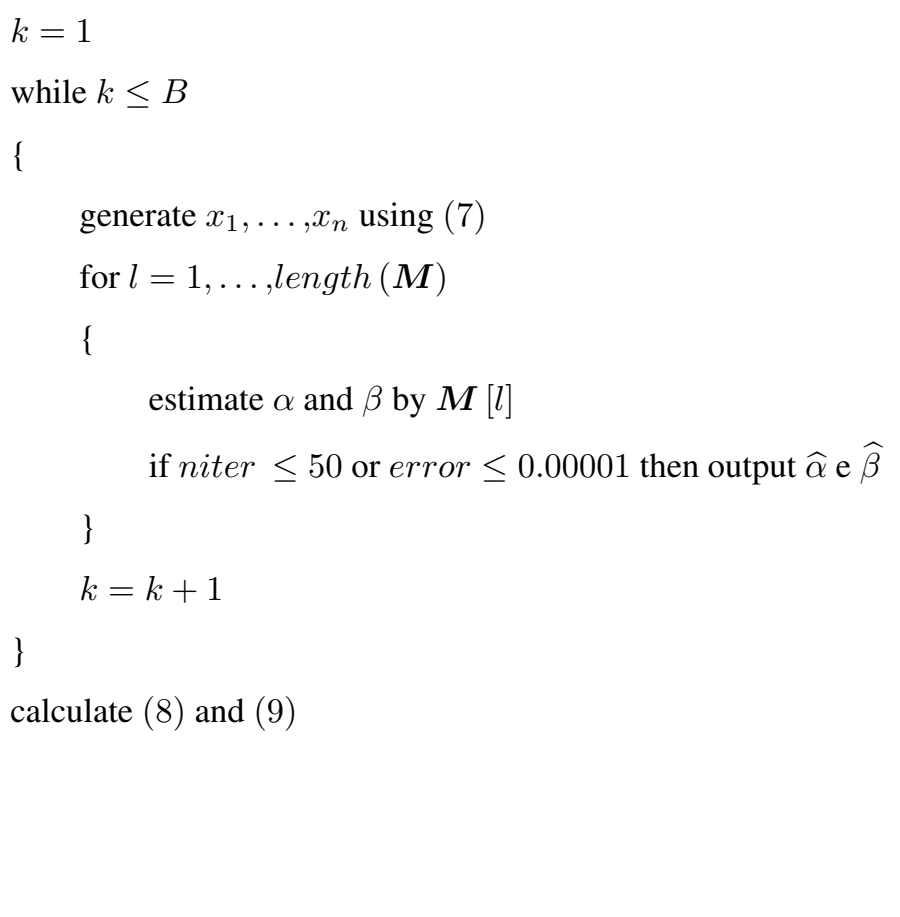

Nas Tabelas 2 à 5 são apresentados os valores de (8) e (9) para cada um dos cenários acima descritos. Os valores sobrescritos indicam o ranque obtido por cada método de estimação. Por exemplo, na Tabela 2, linha 1, tem-se os valores sobrescritos 6, 7, 9 , $1,4,3,5,8$ e 2, demonstrando que no cenário $n=20, \alpha=0.8$ e $\beta=1.0$ o método com menor viés foi o método AD seguido pelo AD2 e assim por diante. A linha "Total" apresenta a soma dos ranques e os respectivos ranques. Por exemplo, na Tabela 2, linha “Total", tem-se os valores 102, 120, 159, 66, 110, 86, 207, 202 e 208 demonstrando que, globalmente, para $\alpha=0.8$ e $\beta=1.0$ o método AD foi "melhor" em termos de viés e acurácia. A fim de tornar mais fácil a visualização destes resultados, na Tabela 6 são apresentados os "Totais" de cada cenário.

Table 2: Estimativas do viés e do erro quadrático médio de $\widehat{\alpha}$ e $\widehat{\beta}(\alpha=0.8$ e $\beta=1.0)$.

\begin{tabular}{|c|c|c|c|c|c|c|c|c|c|c|}
\hline $\mathrm{n}$ & & EMV & CvM & KS & $\mathrm{AD}$ & ADR & ADL & AD2R & AD2L & AD2 \\
\hline \multirow{3}{*}{20} & Viés $(\widehat{\alpha})$ & $0.031^{6}$ & $0.032^{7}$ & $0.035^{9}$ & $0.008^{1}$ & $0.022^{4}$ & $0.018^{3}$ & $0.025^{5}$ & $0.032^{8}$ & $0.014^{2}$ \\
\hline & $\operatorname{EQM}(\widehat{\alpha})$ & $0.009^{3}$ & $0.014^{7}$ & $0.013^{5}$ & $0.008^{1}$ & $0.014^{6}$ & $0.010^{4}$ & $0.029^{9}$ & $0.019^{8}$ & $0.009^{2}$ \\
\hline & Viés $(\beta)$ & $0.011^{6}$ & $0.007^{3}$ & $0.009^{4}$ & $0.010^{5}$ & $0.005^{2}$ & $0.000^{1}$ & $0.018^{8}$ & $0.012^{7}$ & $0.036^{9}$ \\
\hline \multirow{4}{*}{50} & $\operatorname{EQM}(\beta)$ & $0.018^{1}$ & $0.021^{4}$ & $0.021^{5}$ & $0.018^{2}$ & $0.020^{3}$ & $0.021^{6}$ & $0.024^{8}$ & $0.030^{9}$ & $0.022^{7}$ \\
\hline & Viés $(\overline{\widehat{\alpha}})$ & $0.013^{7}$ & $\overline{0} . \overline{0} 2^{6}$ & $\overline{0} . \overline{0} 1 \overline{4}^{8^{-}}$ & $0.00 \overline{4}^{2}$ & $\overline{0.00} 9^{5}$ & $\overline{0} . \overline{00} 7^{4}$ & $\overline{0} . \overline{0} 0 \overline{1}^{-}$ & $0.005^{3}$ & $\overline{0.015^{9}}$ \\
\hline & $\operatorname{EQM}(\widehat{\alpha})$ & $0.003^{1}$ & $0.004^{5}$ & $0.004^{6}$ & $0.003^{2}$ & $0.005^{7}$ & $0.003^{3}$ & $0.010^{9}$ & $0.006^{8}$ & $0.004^{4}$ \\
\hline & Viés $(\beta)$ & $0.006^{7}$ & $0.003^{4}$ & $0.004^{6}$ & $0.003^{3}$ & $0.001^{2}$ & $0.000^{1}$ & $0.011^{8}$ & $0.004^{5}$ & $0.025^{9}$ \\
\hline \multirow[b]{4}{*}{80} & $\operatorname{EQM}(\beta)$ & $0.007^{1}$ & $0.008^{4}$ & $0.008^{6}$ & $0.007^{2}$ & $0.008^{3}$ & $0.008^{5}$ & $0.009^{7}$ & $0.013^{9}$ & $0.010^{8}$ \\
\hline & $\overline{\operatorname{Viés}(\overline{\hat{\alpha}})}$ & $0.008^{7}$ & $\overline{0} . \overline{00} 8^{6}$ & $\overline{0} 0 . \overline{0} 0 \overline{9}^{8^{-}}$ & $0.00 \overline{2}^{2}$ & $0.005^{5}$ & $\overline{0} . \overline{00} 4^{3}$ & $0 . \overline{0} 0 \overline{5}^{4^{-}}$ & $0.000^{T}$ & $\overline{0.014^{9}}$ \\
\hline & $\operatorname{EQM}(\widehat{\alpha})$ & $0.002^{1}$ & $0.003^{5}$ & $0.003^{6}$ & $0.002^{2}$ & $0.003^{7}$ & $0.002^{3}$ & $0.006^{9}$ & $0.004^{8}$ & $0.002^{4}$ \\
\hline & $\operatorname{Viés}(\beta)$ & $0.003^{6}$ & $0.002^{3}$ & $0.002^{5}$ & $0.002^{4}$ & $0.001^{2}$ & $0.000^{1}$ & $0.010^{8}$ & $0.008^{7}$ & $0.022^{9}$ \\
\hline & $\operatorname{EQM}(\beta)$ & $0.004^{1}$ & $0.005^{4}$ & $0.005^{6}$ & $0.005^{2}$ & $0.005^{3}$ & $0.005^{5}$ & $0.006^{7}$ & $0.009^{9}$ & $0.007^{8}$ \\
\hline \multirow{3}{*}{110} & Viés $(\overline{\widehat{\alpha}})$ & $0.005^{6}$ & $\overline{0} .005^{5}$ & $\overline{0} . \overline{0} 0 \overline{6}^{7}$ & $0 . \overline{001} \overline{1}^{\Gamma}$ & $0.004^{4}$ & $\overline{0} .003^{3}$ & $0 . \overline{0} 0 \overline{7}^{8}$ & $0.00 \overline{3}^{2}$ & $0.013^{9}$ \\
\hline & $\operatorname{EQM}(\widehat{\alpha})$ & $0.001^{1}$ & $0.002^{4}$ & $0.002^{6}$ & $0.001^{2}$ & $0.002^{7}$ & $0.001^{3}$ & $0.005^{9}$ & $0.003^{8}$ & $0.002^{5}$ \\
\hline & Viés $(\beta)$ & $0.002^{6}$ & $0.001^{3}$ & $0.002^{4}$ & $0.002^{5}$ & $0.001^{2}$ & $0.000^{1}$ & $0.008^{7}$ & $0.009^{8}$ & $0.019^{9}$ \\
\hline & $\operatorname{EQM}(\beta)$ & $0.003^{1}$ & $0.004^{4}$ & $0.004^{6}$ & $0.003^{2}$ & $0.004^{3}$ & $0.004^{5}$ & $0.004^{7}$ & $0.007^{9}$ & $0.005^{8}$ \\
\hline & $\bar{V}$ Vés $(\overline{\bar{\alpha}})$ & $\overline{0.00} 4^{6}$ & $\overline{0} . \overline{00} 4^{5}-$ & $\overline{0} \overline{0} 0 \overline{5}^{7^{-}}$ & $-0 . \overline{001}\ulcorner$ & $0.003^{3}$ & $\overline{0.00} 2^{2}$ & $\overline{0} . \overline{0} 0 \overline{8}^{8^{-}}$ & $0.0 \overline{3} 4$ & $\overline{0.012^{9}}$ \\
\hline \multirow{2}{*}{140} & $\operatorname{EQM}(\widehat{\alpha})$ & $0.001^{1}$ & $0.001^{4}$ & $0.001^{5}$ & $0.001^{2}$ & $0.002^{7}$ & $0.001^{3}$ & $0.004^{9}$ & $0.002^{8}$ & $0.001^{6}$ \\
\hline & Viés $(\beta)$ & $0.002^{6}$ & $0.001^{4}$ & $0.002^{5}$ & $0.001^{3}$ & $0.000^{2}$ & $0.000^{1}$ & $0.007^{7}$ & $0.009^{8}$ & $0.017^{9}$ \\
\hline & $\mathrm{EQM}(\beta)$ & $0.003^{1}$ & $0.003^{4}$ & $0.003^{5}$ & $0.003^{2}$ & $0.003^{3}$ & $0.003^{6}$ & $0.003^{7}$ & $0.006^{9}$ & $0.004^{8}$ \\
\hline \multirow{3}{*}{170} & $\overline{\operatorname{Viés}}(\overline{\hat{\alpha}})$ & $0.003^{5}$ & $\overline{0.00} 3^{4}$ & $\overline{0} . \overline{0} 0 \overline{4}^{6}$ & $\overline{0.00 \overline{1}^{\top}} \Gamma$ & $\overline{0.002^{3}}$ & $\overline{0.00} 2^{2}$ & $\overline{0} . \overline{0} 0 \overline{8}^{8}$ & $\overline{0.004^{T}}$ & $\overline{0.01} 2^{9}$ \\
\hline & $\operatorname{EQM}(\widehat{\alpha})$ & $0.001^{1}$ & $0.001^{4}$ & $0.001^{5}$ & $0.001^{2}$ & $0.001^{7}$ & $0.001^{3}$ & $0.003^{9}$ & $0.002^{8}$ & $0.001^{6}$ \\
\hline & Viés $(\beta)$ & $0.002^{6}$ & $0.001^{3}$ & $0.001^{4}$ & $0.001^{5}$ & $0.000^{2}$ & $0.000^{1}$ & $0.007^{7}$ & $0.010^{8}$ & $0.016^{9}$ \\
\hline \multirow{4}{*}{200} & $\operatorname{EQM}(\beta)$ & $0.002^{1}$ & $0.002^{4}$ & $0.002^{6}$ & $0.002^{2}$ & $0.002^{3}$ & $0.002^{5}$ & $0.003^{7}$ & $0.005^{9}$ & $0.003^{8}$ \\
\hline & $\overline{\operatorname{Viés}}(\overline{\hat{\alpha}})$ & $0.003^{6}$ & $\overline{0} .002^{4}$ & $\overline{0} 0 . \overline{0} 0 \overline{3}^{5^{-}}$ & $0.000^{\Gamma}$ & $0.0 \overline{0} 2^{3}$ & $\overline{0} . \overline{00} 1^{2}$ & $0 . \overline{0} 0 \overline{8}^{8}$ & $0.00 \overline{5}^{7}$ & $\overline{0.01} 1^{9}$ \\
\hline & $\operatorname{EQM}(\widehat{\alpha})$ & $0.001^{1}$ & $0.001^{4}$ & $0.001^{5}$ & $0.001^{2}$ & $0.001^{6}$ & $0.001^{3}$ & $0.003^{9}$ & $0.002^{8}$ & $0.001^{7}$ \\
\hline & Viés $(\beta)$ & $0.001^{6}$ & $0.001^{2}$ & $0.001^{4}$ & $0.001^{5}$ & $0.001^{3}$ & $0.000^{1}$ & $0.006^{7}$ & $0.009^{8}$ & $0.015^{9}$ \\
\hline & $\operatorname{EQM}(\beta)$ & $0.002^{1}$ & $0.002^{4}$ & $0.002^{5}$ & $0.002^{2}$ & $0.002^{3}$ & $0.002^{6}$ & $0.002^{7}$ & $0.004^{9}$ & $0.003^{8}$ \\
\hline & Total & $\overline{1} 0 \overline{2}^{3}-$ & $-120^{5}$ & $159^{6}$ & $-\overline{6} 6^{\mathrm{T}}-$ & $\overline{1} 1 \overline{0}^{4}$ & $-8 \overline{6}^{-}$ & $207^{8}$ & $\overline{2} 02^{7}-$ & $\overline{2} 0 \overline{8}^{9^{-}}$ \\
\hline
\end{tabular}


Table 3: Estimativas do viés e do erro quadrático médio de $\widehat{\alpha}$ e $\widehat{\beta}(\alpha=0.8$ e $\beta=3.0)$.

\begin{tabular}{|c|c|c|c|c|c|c|c|c|c|c|}
\hline $\mathrm{n}$ & & EMV & CvM & KS & $\mathrm{AD}$ & ADR & ADL & AD2R & AD2L & AD2 \\
\hline \multirow{3}{*}{20} & Viés $(\widehat{\alpha})$ & $0.035^{6}$ & $0.036^{8}$ & $0.039^{9}$ & $0.009^{1}$ & $0.026^{4}$ & $0.020^{3}$ & $0.034^{5}$ & $0.036^{7}$ & $0.014^{2}$ \\
\hline & $\operatorname{EQM}(\widehat{\alpha})$ & $0.011^{3}$ & $0.017^{7}$ & $0.016^{5}$ & $0.010^{1}$ & $0.017^{6}$ & $0.012^{4}$ & $0.039^{9}$ & $0.022^{8}$ & $0.010^{2}$ \\
\hline & Viés $(\beta)$ & $0.003^{1}$ & $0.010^{3}$ & $0.010^{4}$ & $0.015^{5}$ & $0.024^{7}$ & $0.004^{2}$ & $0.087^{9}$ & $0.019^{6}$ & $0.032^{8}$ \\
\hline & $\operatorname{EQM}(\beta)$ & $0.046^{3}$ & $0.050^{6}$ & $0.052^{7}$ & $0.043^{1}$ & $0.050^{5}$ & $0.047^{4}$ & $1.832^{9}$ & $0.060^{8}$ & $0.044^{2}$ \\
\hline \multirow{3}{*}{50} & Viés $(\overline{\widehat{\alpha}})$ & $0.0 \overline{1} 4^{7}$ & $\overline{0} . \overline{01} 4^{6}$ & $0 . \overline{0} 1 \overline{5}^{8}$ & $0 . \overline{0} \overline{4}^{2}$ & $0.011^{5}$ & $\overline{0} .007^{4}$ & $\overline{0} . \overline{0} 0 \overline{4}^{1}$ & $0.0 \overline{6}^{3}$ & $0.016^{9}$ \\
\hline & $\operatorname{EQM}(\widehat{\alpha})$ & $0.003^{1}$ & $0.005^{5}$ & $0.005^{6}$ & $0.004^{2}$ & $0.006^{7}$ & $0.004^{3}$ & $0.011^{9}$ & $0.007^{8}$ & $0.004^{4}$ \\
\hline & Viés $(\beta)$ & $0.001^{2}$ & $0.002^{5}$ & $0.002^{4}$ & $0.004^{6}$ & $0.007^{7}$ & $0.001^{1}$ & $0.016^{8}$ & $0.001^{3}$ & $0.020^{9}$ \\
\hline & $\operatorname{EQM}(\beta)$ & $0.017^{2}$ & $0.018^{4}$ & $0.019^{7}$ & $0.017^{1}$ & $0.018^{3}$ & $0.018^{5}$ & $0.021^{8}$ & $0.024^{9}$ & $0.019^{6}$ \\
\hline & $\overline{V i e ́ s}(\overline{\bar{\alpha}})^{-}$ & $0.0 \overline{0} 9^{6}$ & $\overline{0.009^{7}}$ & $\overline{0} . \overline{0} 1 \overline{0}^{8-}$ & $0 . \overline{003^{2}}$ & $\overline{0.000} 6^{5}$ & $\overline{0} . \overline{00} 5^{4}$ & $\overline{0} . \overline{0} 0 \overline{3}^{3}-$ & $0 . \overline{000^{1}}$ & $\overline{0.01} 5^{9}$ \\
\hline \multirow{2}{*}{80} & $\operatorname{EQM}(\widehat{\alpha})$ & $0.002^{1}$ & $0.003^{5}$ & $0.003^{6}$ & $0.002^{2}$ & $0.003^{7}$ & $0.002^{3}$ & $0.007^{9}$ & $0.004^{8}$ & $0.003^{4}$ \\
\hline & Viés $(\beta)$ & $0.001^{1}$ & $0.002^{4}$ & $0.002^{3}$ & $0.004^{5}$ & $0.006^{7}$ & $0.002^{2}$ & $0.009^{8}$ & $0.005^{6}$ & $0.019^{9}$ \\
\hline & $\operatorname{EQM}(\beta)$ & $0.010^{2}$ & $0.011^{4}$ & $0.012^{6}$ & $0.010^{1}$ & $0.011^{3}$ & $0.011^{5}$ & $0.012^{7}$ & $0.016^{9}$ & $0.012^{8}$ \\
\hline \multirow{3}{*}{110} & $\overline{\operatorname{Viés}}(\overline{\widehat{\alpha}})$ & $0.006^{7}$ & $\overline{0} . \overline{00} 6^{6}$ & $\overline{0} . \overline{0} 0 \overline{6}^{8}$ & $0 . \overline{001} 1^{1-}$ & $0.004^{4}$ & $\overline{0} .003^{3}$ & $\overline{0} . \overline{0} 0 \overline{6}^{5}$ & $0.002^{2}$ & $0.014^{9}$ \\
\hline & $\operatorname{EQM}(\widehat{\alpha})$ & $0.001^{1}$ & $0.002^{5}$ & $0.002^{6}$ & $0.002^{2}$ & $0.002^{7}$ & $0.002^{3}$ & $0.005^{9}$ & $0.003^{8}$ & $0.002^{4}$ \\
\hline & Viés $(\beta)$ & $0.000^{1}$ & $0.001^{3}$ & $0.001^{4}$ & $0.002^{5}$ & $0.003^{6}$ & $0.000^{2}$ & $0.004^{7}$ & $0.006^{8}$ & $0.016^{9}$ \\
\hline & $\operatorname{EQM}(\beta)$ & $0.008^{1}$ & $0.008^{4}$ & $0.008^{6}$ & $0.008^{2}$ & $0.008^{3}$ & $0.008^{5}$ & $0.009^{7}$ & $0.012^{9}$ & $0.009^{8}$ \\
\hline \multirow[b]{3}{*}{140} & $\overline{\operatorname{Viés}}(\overline{\widehat{\alpha}})$ & $0.0 \overline{0} 5^{6}$ & $\overline{0} . \overline{00} 5^{5}$ & $\overline{0} . \overline{0} 0 \overline{5}^{-}$ & $0 . \overline{0} 0 \overline{1}^{1-}$ & $0.0 \overline{0} 3^{4}$ & $\overline{0} . \overline{00} 2^{2}$ & $\overline{0} . \overline{0} 0 \overline{7}^{8}$ & $0.0 \overline{03}^{3}$ & $0.013^{9}$ \\
\hline & $\operatorname{EQM}(\widehat{\alpha})$ & $0.001^{1}$ & $0.002^{4}$ & $0.002^{6}$ & $0.001^{2}$ & $0.002^{7}$ & $0.001^{3}$ & $0.004^{9}$ & $0.002^{8}$ & $0.002^{5}$ \\
\hline & Viés $(\beta)$ & $0.001^{4}$ & $0.000^{2}$ & $0.000^{3}$ & $0.001^{5}$ & $0.002^{7}$ & $0.000^{1}$ & $0.001^{6}$ & $0.007^{8}$ & $0.014^{9}$ \\
\hline & $\operatorname{EQM}(\beta)$ & $0.006^{1}$ & $0.006^{4}$ & $0.006^{6}$ & $0.006^{2}$ & $0.006^{3}$ & $0.006^{5}$ & $0.007^{7}$ & $0.010^{9}$ & $0.007^{8}$ \\
\hline \multirow{3}{*}{170} & $\overline{V i e ́ s}(\overline{\hat{\alpha}})$ & $0.0 \overline{0} 4^{5}$ & $\overline{0.00} 4^{4}$ & $\overline{0} . \overline{0} 0 \overline{4}^{6-}$ & $0.001^{1-}$ & $0.003^{3}$ & $\overline{0} . \overline{00} 2^{2}$ & $\overline{0} . \overline{0} 0 \overline{8}^{8}$ & $0.004^{7}$ & $0.013^{9}$ \\
\hline & $\operatorname{EQM}(\widehat{\alpha})$ & $0.001^{1}$ & $0.001^{4}$ & $0.001^{6}$ & $0.001^{2}$ & $0.001^{7}$ & $0.001^{3}$ & $0.004^{9}$ & $0.002^{8}$ & $0.001^{5}$ \\
\hline & Viés $(\beta)$ & $0.000^{3}$ & $0.001^{4}$ & $0.001^{5}$ & $0.001^{6}$ & $0.002^{7}$ & $0.000^{1}$ & $0.000^{2}$ & $0.008^{8}$ & $0.013^{9}$ \\
\hline & $\operatorname{EQM}(\beta)$ & $0.005^{1}$ & $0.005^{4}$ & $0.005^{6}$ & $0.005^{2}$ & $0.005^{3}$ & $0.005^{5}$ & $0.006^{7}$ & $0.008^{9}$ & $0.006^{8}$ \\
\hline & $\overline{\operatorname{Viess}}(\overline{\widehat{\alpha}})$ & $\overline{0.003^{6}}$ & $\overline{0} . \overline{00} 3^{4}$ & $\overline{0} . \overline{0} 0 \overline{3}^{5}$ & $0 . \overline{0} 0 \overline{1}^{-}$ & $\overline{0.00} 2^{3}$ & $\overline{0} . \overline{00} 2^{2}$ & $\overline{0} . \overline{0} 0 \overline{8}^{8}$ & $0 . \overline{0} \overline{4} 7$ & $\overline{0.01} 2^{9}$ \\
\hline \multirow{3}{*}{200} & $\operatorname{EQM}(\widehat{\alpha})$ & $0.001^{1}$ & $0.001^{4}$ & $0.001^{5}$ & $0.001^{2}$ & $0.001^{7}$ & $0.001^{3}$ & $0.003^{9}$ & $0.002^{8}$ & $0.001^{6}$ \\
\hline & Viés $(\beta)$ & $0.001^{4}$ & $0.000^{1}$ & $0.000^{2}$ & $0.001^{6}$ & $0.002^{7}$ & $0.000^{3}$ & $0.001^{5}$ & $0.008^{8}$ & $0.012^{9}$ \\
\hline & $\operatorname{EQM}(\beta)$ & $0.004^{1}$ & $0.004^{4}$ & $0.004^{6}$ & $0.004^{2}$ & $0.004^{3}$ & $0.004^{5}$ & $0.005^{7}$ & $0.007^{9}$ & $0.005^{8}$ \\
\hline & Iotal & 19 & $120^{2}$ & $100^{\circ}$ & $/ 1+$ & $14 / 0$ & & $-1 \overline{9} 8^{9}$ & $\overline{19} 5^{7}$ & $196^{8}$ \\
\hline
\end{tabular}

Table 4: Estimativas do viés e do erro quadrático médio de $\widehat{\alpha}$ e $\widehat{\beta}(\alpha=2.0$ e $\beta=0.8)$.

\begin{tabular}{|c|c|c|c|c|c|c|c|c|c|c|}
\hline $\mathrm{n}$ & & EMV & CvM & KS & $\mathrm{AD}$ & ADR & ADL & AD2R & AD2L & AD2 \\
\hline \multirow{3}{*}{20} & Viés $(\widehat{\alpha})$ & $0.062^{6}$ & $0.064^{7}$ & $0.070^{9}$ & $0.016^{1}$ & $0.043^{4}$ & $0.035^{3}$ & $0.050^{5}$ & $0.065^{8}$ & $0.029^{2}$ \\
\hline & $\operatorname{EQM}(\widehat{\alpha})$ & $0.036^{3}$ & $0.055^{7}$ & $0.053^{5}$ & $0.033^{1}$ & $0.054^{6}$ & $0.040^{4}$ & $0.117^{9}$ & $0.076^{8}$ & $0.035^{2}$ \\
\hline & Viés $(\beta)$ & $0.011^{6}$ & $0.007^{3}$ & $0.009^{4}$ & $0.010^{5}$ & $0.005^{2}$ & $0.000^{1}$ & $0.018^{8}$ & $0.012^{7}$ & $0.036^{9}$ \\
\hline \multirow[b]{4}{*}{50} & $\operatorname{EQM}(\beta)$ & $0.018^{1}$ & $0.021^{4}$ & $0.021^{5}$ & $0.018^{2}$ & $0.020^{3}$ & $0.021^{6}$ & $0.024^{8}$ & $0.030^{9}$ & $0.022^{7}$ \\
\hline & $\overline{V i e ́ s}(\widehat{\widehat{\alpha}})$ & $0.025^{7}$ & $\overline{0.024^{6}}$ & $\overline{0} . \overline{0} 2 \overline{7}^{8^{-}}$ & $0.007^{2}$ & $0.018^{5}$ & $\overline{0} . \overline{01} 3^{4}$ & $\overline{0} . \overline{0} 0 \overline{2}^{-}$ & $0.010^{3}$ & $\overline{0.031^{9}}$ \\
\hline & $\operatorname{EQM}(\widehat{\alpha})$ & $0.012^{1}$ & $0.017^{5}$ & $0.018^{6}$ & $0.012^{2}$ & $0.018^{7}$ & $0.014^{3}$ & $0.039^{9}$ & $0.024^{8}$ & $0.014^{4}$ \\
\hline & Viés $(\beta)$ & $0.006^{7}$ & $0.003^{4}$ & $0.005^{6}$ & $0.003^{3}$ & $0.001^{2}$ & $0.000^{1}$ & $0.011^{8}$ & $0.004^{5}$ & $0.025^{9}$ \\
\hline & $\operatorname{EQM}(\beta)$ & $0.007^{1}$ & $0.008^{4}$ & $0.008^{6}$ & $0.007^{2}$ & $0.008^{3}$ & $0.008^{5}$ & $0.009^{7}$ & $0.013^{9}$ & $0.010^{8}$ \\
\hline \multirow{3}{*}{80} & $\overline{\operatorname{Viés}}(\overline{\widehat{\alpha}})$ & $\overline{0.016^{7}}$ & $\overline{0} . \overline{01} \overline{6}^{6}$ & $\overline{0} . \overline{0} 1 \overline{7}^{8}$ & $0 . \overline{0} \overline{5}^{2}$ & $0.011^{5}$ & $\overline{0} . \overline{00} 9^{3}$ & $\overline{0} . \overline{0} 1 \overline{0}^{4}$ & $0.00^{1}$ & $\overline{0.0} \overline{2} 9^{\overline{9}}$ \\
\hline & $\operatorname{EQM}(\widehat{\alpha})$ & $0.007^{1}$ & $0.010^{5}$ & $0.010^{6}$ & $0.007^{2}$ & $0.011^{7}$ & $0.008^{3}$ & $0.025^{9}$ & $0.014^{8}$ & $0.009^{4}$ \\
\hline & Viés $(\beta)$ & $0.003^{6}$ & $0.002^{3}$ & $0.002^{5}$ & $0.002^{4}$ & $0.001^{2}$ & $0.000^{1}$ & $0.010^{8}$ & $0.008^{7}$ & $0.022^{9}$ \\
\hline & $\operatorname{EQM}(\beta)$ & $0.004^{1}$ & $0.005^{4}$ & $0.005^{5}$ & $0.005^{2}$ & $0.005^{3}$ & $0.005^{6}$ & $0.006^{7}$ & $0.009^{9}$ & $0.007^{8}$ \\
\hline \multirow{3}{*}{110} & $\overline{\operatorname{Viés}}(\overline{\widehat{\alpha}})$ & $0.0 \overline{1} 1^{6}$ & $\overline{0} . \overline{01} 0^{5}$ & $\overline{0} . \overline{0} 1 \overline{1}^{-}$ & $0 . \overline{00} \overline{3}^{1-}$ & $\overline{0.000} 7^{4}$ & $\overline{0} . \overline{00} 6^{3}$ & $\overline{0} . \overline{0} 1 \overline{3}^{8-}$ & $0.005^{2}$ & $\overline{0.02} 7^{9}$ \\
\hline & $\operatorname{EQM}(\widehat{\alpha})$ & $0.005^{1}$ & $0.007^{4}$ & $0.007^{6}$ & $0.005^{2}$ & $0.008^{7}$ & $0.006^{3}$ & $0.019^{9}$ & $0.011^{8}$ & $0.007^{5}$ \\
\hline & Viés $(\beta)$ & $0.002^{6}$ & $0.001^{3}$ & $0.002^{5}$ & $0.002^{4}$ & $0.001^{2}$ & $0.000^{1}$ & $0.008^{7}$ & $0.009^{8}$ & $0.019^{9}$ \\
\hline \multirow{4}{*}{140} & $\operatorname{EQM}(\beta)$ & $0.003^{1}$ & $0.004^{4}$ & $0.004^{6}$ & $0.003^{2}$ & $0.004^{3}$ & $0.004^{5}$ & $0.004^{7}$ & $0.007^{9}$ & $0.005^{8}$ \\
\hline & $\bar{V}$ iés $(\widehat{\widehat{\alpha}})$ & $\overline{0.009^{6}}$ & $\overline{0} . \overline{00} 8^{5}$ & $\overline{0} . \overline{0} 0 \overline{9}^{-}$ & $0.002^{1-}$ & $0.006^{3}$ & $\overline{0} . \overline{00} 4^{2}$ & $\overline{0} . \overline{0} 1 \overline{5}^{8}$ & $0.0 \overline{0} 7^{4}$ & $0.0 \overline{2} 5^{\overline{9}}$ \\
\hline & $\operatorname{EQM}(\widehat{\alpha})$ & $0.004^{1}$ & $0.005^{4}$ & $0.006^{5}$ & $0.004^{2}$ & $0.006^{7}$ & $0.005^{3}$ & $0.015^{9}$ & $0.009^{8}$ & $0.006^{6}$ \\
\hline & Viés $(\beta)$ & $0.002^{6}$ & $0.001^{4}$ & $0.002^{5}$ & $0.001^{3}$ & $0.000^{2}$ & $0.000^{1}$ & $0.007^{7}$ & $0.009^{8}$ & $0.017^{9}$ \\
\hline & $\operatorname{EQM}(\beta)$ & $0.003^{1}$ & $0.003^{4}$ & $0.003^{5}$ & $0.003^{2}$ & $0.003^{3}$ & $0.003^{6}$ & $0.003^{7}$ & $0.006^{9}$ & $0.004^{8}$ \\
\hline \multirow{3}{*}{170} & Viés $(\overline{\widehat{\alpha}})$ & $\overline{0.007^{5}}$ & $\overline{0.00} 6^{4}$ & $\overline{0} . \overline{0} 0 \overline{7}^{6}$ & $0 . \overline{0} \overline{01}{ }^{1-}$ & $\overline{0.00} 4^{3}$ & $\overline{0} . \overline{00} 3^{2}$ & $\overline{0} \overline{0} 1 \overline{6}^{8-}$ & $0.00 \overline{9}^{7}$ & $0.024^{9}$ \\
\hline & $\operatorname{EQM}(\widehat{\alpha})$ & $0.003^{1}$ & $0.004^{4}$ & $0.005^{5}$ & $0.003^{2}$ & $0.005^{7}$ & $0.004^{3}$ & $0.013^{9}$ & $0.007^{8}$ & $0.005^{6}$ \\
\hline & & $0.002^{6}$ & $0.001^{3}$ & $0.001^{4}$ & $0.001^{5}$ & $0.000^{2}$ & $0.000^{1}$ & $0.007^{7}$ & $0.010^{8}$ & $0.016^{9}$ \\
\hline & $\operatorname{EQM}(\beta)$ & $0.002^{1}$ & $0.002^{4}$ & $0.002^{6}$ & $0.002^{2}$ & $0.002^{3}$ & $0.002^{5}$ & $0.003^{7}$ & $0.005^{9}$ & $0.003^{8}$ \\
\hline & & $\overline{0.00} 6^{6}$ & $\overline{0} . \overline{00} 5^{4}$ & $\overline{0} . \overline{0} 0 \overline{6}^{-}$ & $\overline{0} \overline{0} \overline{0} 1^{1-}$ & $\overline{0.00} 3^{3}$ & $\overline{0} . \overline{00} 3^{2}$ & $\overline{0} . \overline{0} 1 \overline{7}^{-}$ & $0 . \overline{009} \overline{9}^{7}$ & $\overline{0.02} 3^{9}$ \\
\hline \multirow{4}{*}{200} & $\operatorname{EQM}(\widehat{\alpha})$ & $0.003^{1}$ & $0.004^{4}$ & $0.004^{5}$ & $0.003^{2}$ & $0.004^{6}$ & $0.003^{3}$ & $0.011^{9}$ & $0.006^{8}$ & $0.004^{7}$ \\
\hline & Viés $(\beta)$ & $0.001^{6}$ & $0.001^{2}$ & $0.001^{4}$ & $0.001^{5}$ & $0.001^{3}$ & $0.000^{1}$ & $0.006^{7}$ & $0.009^{8}$ & $0.015^{9}$ \\
\hline & $\operatorname{EQM}(\beta)$ & $0.002^{1}$ & $0.002^{4}$ & $0.002^{5}$ & $0.002^{2}$ & $0.002^{3}$ & $0.002^{6}$ & $0.002^{7}$ & $0.004^{9}$ & $0.003^{8}$ \\
\hline & Total & $\overline{1} 0 \overline{2}^{3^{-}}$ & $-1 \overline{20} 0^{5}$ & $1 \overline{5} 9^{6}$ & & $\overline{1} 1 \overline{0}^{4^{-}}$ & $-8 \overline{7}^{-}$ & $2 \overline{0} 7^{\overline{8}}$ & $\overline{2} 0 \overline{2}^{7}-$ & $\overline{2} 0 \overline{8}^{9^{-}}$ \\
\hline
\end{tabular}


Table 5: Estimativas do viés e do erro quadrático médio de $\widehat{\alpha}$ e $\widehat{\beta}(\alpha=2.0$ e $\beta=3.0)$.

\begin{tabular}{|c|c|c|c|c|c|c|c|c|c|c|}
\hline $\mathrm{n}$ & & EMV & CvM & KS & $\mathrm{AD}$ & ADR & ADL & $2 \mathrm{R}$ & D2L & \\
\hline \multirow{4}{*}{20} & Viés $(\widehat{\alpha})$ & $0.070^{6}$ & $0.073^{8}$ & $0.079^{9}$ & $0.019^{1}$ & $0.051^{4}$ & $0.039^{3}$ & $.067^{5}$ & $0.072^{7}$ & $0.028^{2}$ \\
\hline & EQM & $043^{3}$ & $068^{7}$ & $066^{5}$ & $038^{1}$ & $.067^{6}$ & $.047^{4}$ & $.154^{9}$ & $0.089^{8}$ & $0.040^{2}$ \\
\hline & és( & $003^{1}$ & $010^{3}$ & $011^{4}$ & $015^{5}$ & $0.024^{7}$ & $0.004^{2}$ & $087^{9}$ & $0.019^{6}$ & $0.032^{8}$ \\
\hline & $\mathrm{EQM}($ & $0.046^{3}$ & $0.050^{6}$ & $0.052^{7}$ & $0.043^{1}$ & $0.050^{5}$ & $0.047^{4}$ & $1.833^{9}$ & $0.060^{8}$ & $.044^{2}$ \\
\hline & $\overline{\text { Viés }}$ & $0.02 \overline{2} 8^{7}$ & $\overline{0} . \overline{0} 7^{6}$ & $\overline{0} . \overline{0} 3 \overline{1}^{8}$ & $0 . \overline{0} \overline{8}^{2}$ & $\overline{0.0} \overline{2} 1^{5}$ & $\overline{0} . \overline{01} 5^{4}$ & $\overline{0} . \overline{0} 0 \overline{7}^{1}$ & $0.013^{3}$ & $\overline{0.03} 1^{9}$ \\
\hline \multirow{2}{*}{50} & $\mathrm{EQM}$ & $0.014^{1}$ & $0.021^{5}$ & $0.021^{6}$ & $0.014^{2}$ & $0.022^{7}$ & $0.016^{3}$ & $0.046^{9}$ & & $0.016^{4}$ \\
\hline & Viés & 2 & $002^{5}$ & $002^{4}$ & $0.004^{6}$ & $0.007^{7}$ & $0.001^{1}$ & $0.016^{8}$ & $0.001^{3}$ & $0.020^{9}$ \\
\hline & $\mathrm{EQM}($ & $0.017^{2}$ & $0.018^{4}$ & $0.019^{7}$ & $0.017^{1}$ & $0.018^{3}$ & $0.018^{5}$ & $0.021^{8}$ & $0.024^{9}$ & $0.019^{6}$ \\
\hline \multirow{3}{*}{80} & $\overline{\operatorname{Viés}}(\overline{\hat{a}}$ & $0.018^{6}$ & $\overline{0} . \overline{01} \overline{8}^{7}$ & $\overline{0} . \overline{0} 1 \overline{9}^{8}$ & $0.00 \overline{5}^{2}$ & $\overline{0.013^{5}}$ & $\overline{0} . \overline{01} \overline{0}^{4}$ & & $0.00 \overline{1}^{-}$ & $\overline{0.03} 0^{9}$ \\
\hline & $\mathrm{EQM}($ & $3^{1}$ & $0.012^{5}$ & $0.012^{6}$ & $0.009^{2}$ & $0.013^{7}$ & $0.009^{3}$ & $0.029^{9}$ & $0.016^{8}$ & $0.011^{4}$ \\
\hline & V & 0 & & $0.002^{4}$ & $0.004^{5}$ & 0.00 & $0.002^{2}$ & $0.009^{8}$ & & $0.019^{9}$ \\
\hline & EQN & 0 & $1^{4}$ & $0.012^{6}$ & $0.010^{1}$ & 0.01 & 0.01 & $012^{7}$ & $0.016^{9}$ & $12^{8}$ \\
\hline & & 0.01 & $\overline{0} . \overline{01} 1^{6}$ & $\overline{0} . \overline{0} 1 \overline{3}^{8}$ & $0 . \overline{0} \overline{3}^{\top}$ & $\overline{0.0 \overline{9} 9^{4}}$ & $\overline{0} . \overline{00} \overline{6}^{3}$ & $\overline{0} . \overline{0} 1 \overline{1}^{5-}$ & $0.005^{2}$ & $0.0 \overline{2} 8^{9}$ \\
\hline \multirow{3}{*}{110} & EQN & $006^{1}$ & $0.008^{5}$ & $0.009^{6}$ & $0.006^{2}$ & $0.009^{7}$ & $0.007^{3}$ & $0.022^{9}$ & $0.012^{8}$ & $0.008^{4}$ \\
\hline & & & 0 & $001^{4}$ & $0.002^{5}$ & & & $0.004^{7}$ & $0.006^{8}$ & $0.016^{9}$ \\
\hline & EQM( & 00 & 0084 & $0.008^{6}$ & $0.008^{2}$ & & $0.008^{5}$ & $0.009^{7}$ & $0.012^{9}$ & $0.009^{8}$ \\
\hline \multirow{5}{*}{140} & Viés $(\overline{\hat{\alpha}})$ & $0.0 \overline{1}$ & $\overline{0} . \overline{00}$ & $\overline{0} . \overline{0} 1 \overline{0}^{7}$ & $0 . \overline{00} 2^{1}$ & $\overline{0.007^{4}}$ & $\overline{0} . \overline{00}$ & $\overline{0} . \overline{0} 1 \overline{4}^{8}$ & $0.00 \overline{7}^{3}$ & $\overline{0.0} 0 \overline{2} 6^{9}$ \\
\hline & & $1^{1}$ & $006^{4}$ & $0.007^{6}$ & $0.005^{2}$ & 0.0 & 0.00 & $0.017^{9}$ & $0.010^{8}$ & $0.006^{5}$ \\
\hline & & & & $000^{3}$ & $0.001^{5}$ & 0.0 & 0.06 & & $0.007^{8}$ & $0.014^{9}$ \\
\hline & EQI & 0.00 & & $0.006^{6}$ & $0.006^{2}$ & & & & & $0.007^{8}$ \\
\hline & & $\overline{0.00} 8^{5}$ & & $\overline{0} . \overline{0} 0 \overline{8}^{6}$ & $\overline{0.00} \overline{2}^{1}$ & $\overline{0.00} 5^{3}$ & $\overline{0.00} \overline{4}^{2}$ & $\overline{0} . \overline{0} 1 \overline{5}^{8}$ & $\overline{0.00 \overline{9}^{\top}}$ & $\overline{0.02} 5^{9}$ \\
\hline \multirow{3}{*}{170} & E & $0.004^{1}$ & $0.005^{4}$ & $0.006^{6}$ & $0.004^{2}$ & $0.006^{7}$ & $0.004^{3}$ & $0.015^{9}$ & $0.008^{8}$ & $0.005^{5}$ \\
\hline & & & & $0.001^{5}$ & & & & & & $0.013^{9}$ \\
\hline & $\operatorname{EQM}(\beta)$ & & & $0.005^{6}$ & $0.005^{2}$ & & $0.005^{5}$ & $0.006^{7}$ & $0.008^{9}$ & $0.006^{8}$ \\
\hline \multirow{5}{*}{200} & & & & $\overline{0} . \overline{0} 0 \overline{6}^{5}$ & $0 . \overline{001} \overline{1}^{\top}$ & 0.00 & $\overline{0} . \overline{00} 3^{2}$ & $\overline{0} . \overline{0} 1 \overline{6}^{8}$ & $0.00 \overline{9}^{\top}$ & $0.0 \overline{2} 4^{9}$ \\
\hline & & & & & & & & & & $0.005^{6}$ \\
\hline & & & & & & & & & & $0.012^{9}$ \\
\hline & & $0.004^{1}$ & $0.004^{4}$ & $0.004^{6}$ & $0.004^{2}$ & $0.004^{3}$ & $0.004^{5}$ & $0.005^{7}$ & $0.007^{9}$ & $0.005^{8}$ \\
\hline & Total & $\overline{7} \overline{9^{2}}-$ & ${ }^{-} 1 \overline{25^{4}}$ & $1 \overline{6} 2^{6}$ & $-F$ & $\overline{1} 4 \overline{7}^{5^{-}}$ & $-8 \overline{7}^{3^{-}}$ & $1 \overline{9} 8^{9}$ & $195^{7}$ & $\overline{196^{8}}$ \\
\hline
\end{tabular}

Table 6: Performance geral dos métodos de estimação segundo a magnitude do viés e do erro quadrático médio (base: soma dos ranques, expoente: ranque).

\begin{tabular}{cccccccccc}
\hline & \multicolumn{8}{c}{ Método de estimação } \\
\cline { 2 - 9 } Cenário & EMV & CvM & KS & AD & ADR & ADL & AD2R & AD2L & AD2 \\
\hline 1 & $102^{3}$ & $120^{5}$ & $159^{6}$ & $66^{1}$ & $110^{4}$ & $86^{2}$ & $207^{8}$ & $202^{7}$ & $208^{9}$ \\
2 & $79^{2}$ & $126^{4}$ & $160^{6}$ & $71^{1}$ & $147^{5}$ & $88^{3}$ & $198^{9}$ & $195^{7}$ & $196^{8}$ \\
3 & $102^{3}$ & $120^{5}$ & $159^{6}$ & $65^{1}$ & $110^{4}$ & $87^{2}$ & $207^{8}$ & $202^{7}$ & $208^{9}$ \\
4 & $79^{2}$ & $125^{4}$ & $162^{6}$ & $71^{1}$ & $147^{5}$ & $87^{3}$ & $198^{9}$ & $195^{7}$ & $196^{8}$ \\
\hline Total & $362^{3}$ & $491^{4}$ & $640^{6}$ & $273^{1}$ & $514^{5}$ & $348^{2}$ & $810^{9}$ & $794^{7}$ & $808^{8}$ \\
\hline
\end{tabular}

A partir de todos os resultados apresentados anteriormente tem-se evidências de que o melhor método de estimação dos parâmetros da distribuição Lindley potência inversa, em termos do viés e do erro quadrático médio, independente dos cenários, é o método baseado na estatística do teste de Anderson-Darling. Na sequência tem-se o ADL e o método da máxima verossimilhança. Em todos os métodos, como esperado, o viés e o erro quadrático médio tendem a zero a medida que o tamanho da amostra aumenta. É interessante observar que diferentes valores de $\alpha$ não alteram os ranques dos métodos. A troca de posições entre os métodos acontece apenas quando se varia o valor de $\beta$.

\section{Aplicações}

Nesta seção são analisados dois conjuntos de dados retirados da literatura. O primeiro conjunto foi utilizado por Nadarajah et al. (2011) em seu trabalho sobre a distribuição Lindley generalizada enquanto que o segundo foi utilizado por Mahmoud e Mandouh (2013) em seu trabalho sobre a distribuição Fréchet transmutada.

As estimativas de $\alpha$ e $\beta$ obtidas por todos os métodos estão apresentadas na Tabela 7. No apêndice é apresentado o código do ambiente $\mathrm{R}$ usado para obter as estimativas. 
Table 7: Estimativas dos parâmetros considerando os diferentes métodos de estimação.

\begin{tabular}{ccc|cc}
\hline & \multicolumn{2}{c|}{ Dados I } & \multicolumn{2}{c}{ Dados II } \\
\hline Método & $\widehat{\alpha}$ & $\widehat{\beta}$ & $\widehat{\alpha}$ & $\widehat{\beta}$ \\
\hline EMV & 3.9812 & 6.7190 & 5.3904 & 7.1998 \\
CvM & 4.2344 & 7.6697 & 5.8745 & 8.7244 \\
KS & 4.5445 & 9.0261 & 5.7193 & 8.1423 \\
AD & 3.9518 & 6.6767 & 5.6929 & 8.0483 \\
ADR & 4.0640 & 7.0508 & 6.0375 & 9.2310 \\
ADL & 3.8497 & 6.4498 & 5.4272 & 7.4697 \\
AD2R & 3.8254 & 6.1679 & 4.8084 & 5.4120 \\
AD2L & 3.5941 & 5.8952 & 4.5956 & 5.9461 \\
AD2 & 3.7771 & 6.0858 & 4.9072 & 6.0946 \\
\hline
\end{tabular}

Na Tabela 8 são apresentadas os valores das estatísticas dos testes de aderência de Kolmogorov-Smirnov (KS), AndersonDarling (AD) e Cramér-von Mises (CvM) e os seus respectivos valores- $p$. É importante notar que não existem evidências em não se usar a distribuição Lindley potência inversa na análise de ambos os conjuntos de dados.

Table 8: Resultados da aplicação dos testes de aderência.

\begin{tabular}{ccccccc}
\hline \multicolumn{7}{c}{ Dados I } \\
\hline Método & KS & valor $-p$ & AD & valor $-p$ & CvM & valor- $p$ \\
\hline EMV & 0.1031 & 0.9837 & 0.1560 & 0.9982 & 0.0269 & 0.9874 \\
CvM & 0.0929 & 0.9952 & 0.1827 & 0.9946 & 0.0247 & 0.9918 \\
KS & 0.0810 & 0.9994 & 0.2816 & 0.9508 & 0.0274 & 0.9860 \\
AD & 0.1006 & 0.9875 & 0.1554 & 0.9983 & 0.0269 & 0.9872 \\
ADR & 0.0977 & 0.9910 & 0.1597 & 0.9979 & 0.0255 & 0.9903 \\
ADL & 0.0971 & 0.9917 & 0.1592 & 0.9979 & 0.0287 & 0.9827 \\
AD2R & 0.1113 & 0.9653 & 0.1671 & 0.9971 & 0.0311 & 0.9753 \\
AD2L & 0.1091 & 0.9712 & 0.2029 & 0.9898 & 0.0382 & 0.9462 \\
AD2 & 0.1084 & 0.9727 & 0.1684 & 0.9969 & 0.0314 & 0.9743 \\
\hline \multicolumn{7}{c}{ Dados II } \\
\hline Método & KS & valor- $p$ & AD & valor- $-p$ & CvM & valor- $p$ \\
\hline EMV & 0.0780 & 0.8091 & 0.5389 & 0.7069 & 0.0714 & 0.7445 \\
CvM & 0.0772 & 0.8194 & 0.5199 & 0.7259 & 0.0550 & 0.8474 \\
KS & 0.0663 & 0.9276 & 0.4926 & 0.7537 & 0.0574 & 0.8321 \\
AD & 0.0673 & 0.9195 & 0.4920 & 0.7543 & 0.0583 & 0.8265 \\
ADR & 0.0828 & 0.7498 & 0.5618 & 0.6842 & 0.0564 & 0.8381 \\
ADL & 0.0808 & 0.7755 & 0.5308 & 0.7150 & 0.0670 & 0.7721 \\
AD2R & 0.1290 & 0.2250 & 1.2233 & 0.2587 & 0.2006 & 0.2668 \\
AD2L & 0.1317 & 0.2059 & 1.2145 & 0.2620 & 0.1967 & 0.2743 \\
AD2 & 0.0990 & 0.5345 & 0.7995 & 0.4809 & 0.1167 & 0.5104 \\
\hline
\end{tabular}

\section{Conclusão}

Neste artigo foi estudada a performance de oito métodos de estimação baseados nas estatísticas usadas na avaliação da qualidade do ajuste, em contrapartida ao método da máxima verossimilhança. Por meio de simulações verificamos, de forma geral, que o método de Anderson-Darling gerou os menores viéses e erros quadráticos médios, seguido pelos métodos da máxima verossimilhança e ADL. Ao ser aplicado em dois conjuntos de dados reais, os critérios de comparação apontaram o método de Anderson-Darling como o que forneceu o melhor ajuste aos dados, seguido pelo método da máxima verossimilhança. Desta forma, tem-se que os métodos de Anderson-Darling e sua variação ADL podem ser uma alternativa na estimação de parâmetros da distribuição Lindley potência inversa, visto sua qualidade de ajuste e os baixos valores do viés e do erro quadrático médio.

\section{Agradecimentos}

Os autores agradecem os revisores pelas valiosas sugestões. 


\section{Apêndice}

Neste apêndice é apresentado o código R (R Core Team, 2017) utilizado para obter as estimativas dos parâmetros pelos nove métodos discutidos no artigo. A título de ilustração considera-se somente o segundo conjunto de dados, retirado de Mahmoud e Mandouh (2013).

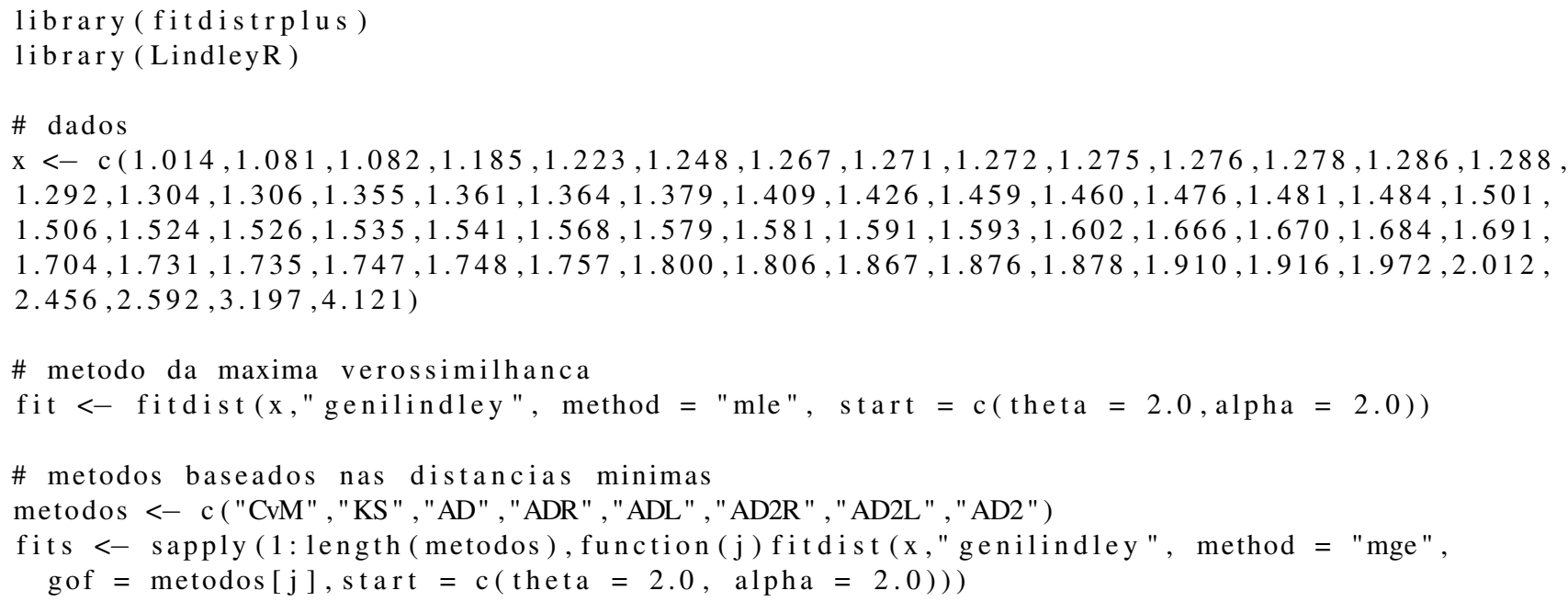

\section{Referências}

Barco, K. V. P., Mazucheli, J., Janeiro, V. (2017). The inverse power Lindley distribution. Communications in Statistics - Simulation and Computation, 46(8), 6308-6323.

D’Agostino, R. B., Stephens, M. A. (1986). Goodness-of-Fit Techniques. Taylor \& Francis.

Dey, S., Mazucheli, J., Nadarajah, S. (2017). Kumaraswamy distribution: Different methods of estimation. Computational and Applied Mathematics, pp. 1-18.

Doornik, J. A. (2007). Object-Oriented Matrix Programming Using Ox, 3rd ed. London: Timberlake Consultants Press and Oxford.

do Espirito-Santo, A. P. J., Mazucheli, J. (2015). Comparison of estimation methods for the Marshall-Olkin extended Lindley distribution. Journal of Statistical Computation and Simulation, 85(17), 3437-3450.

Ghitany, M. E., Atieh, B., Nadarajah, S. (2008). Lindley distribution and its application. Mathematics and Computers in Simulation, 78(4), 493-506.

Ghitany, M. E., Al-Mutairi, D. K., Balakrishnan, N., Al-Enezi, L. J. (2013). Power Lindley distribution and associated inference. Computational Statistics and Data Analysis, 64, 20-33.

Gupta, R. D., Kundu, D. (2001). Generalized Exponential distribution: Different method of estimations. Journal of Statistical Computation and Simulation, 69(4), 315-337.

Kundu, D., Raqab, M. Z. (2005). Generalized Rayleigh distribution: Different methods of estimations. Computational Statistics \& Data Analysis, 49(1), 187-200.

Lehmann, E. J., Casella, G. (1998). Theory of Point Estimation. Springer Verlag.

Lindley, D. V. (1958). Fiducial distributions and Bayes' theorem. Journal of the Royal Statistical Society, $20(1), 102-107$.

Lucenõ, A. (2006). Fitting the Generalized Pareto distribution to data using maximum goodness-of-fit estimators. Computational Statistics \& Data Analysis, 51(2), 904-917.

Mahmoud, M. R., Mandouh, R. M. (2013). On the transmuted Fréchet distribution. Journal of Applied Sciences Research, 9(10), 5553-5561. 
Mazucheli, J., Louzada, F., Ghitany, M. E. (2013). Comparison of estimation methods for the parameters of the weighted Lindley distribution. Applied Mathematics and Computation, 220, 463-471.

Mazucheli, J., Fernandes, L. B., de Oliveira, R. P. (2016). LindleyR: The Lindley Distribution and Its Modifications. URL https://CRAN.R-project.org/package=LindleyR, R package version 1.1.0.

Mazucheli, J., Ghitany, M. E., Louzada, F. (2017). Comparisons of ten estimation methods for the parameters of Marshall-Olkin extended Exponential distribution. Communications in Statistics - Simulation and Computation, 46(7), 5627-5645.

Nadarajah, S., Bakouch, H. S., Tahmasbi, R. (2011). A generalized Lindley distribution. Sankhya B, 73(2), 331-359.

Pawitan, Y. (2001). In All Likelihood: Statistical Modelling and Inference Using Likelihood. Oxford University Press, Oxford.

R Core Team (2017). R: A Language and Environment for Statistical Computing. R Foundation for Statistical Computing, Vienna, Austria, URL https: / / www.R-project.org/.

Rohde, C. A. (2014). Introductory Statistical Inference with the Likelihood Function. Springer-Verlag, New York.

Sharma, V. K., Singh, S. K., Singh, U., Agiwal, V. (2015a). The inverse Lindley distribution: A stress-strength reliability model with application to head and neck cancer data. Journal of Industrial and Production Engineering, 32(3), 162-173.

Sharma, V. K., Singh, S. K., Singh, U., Merovci, F. (2015b). The generalized inverse Lindley distribution: A new inverse statistical model for the study of upside-down bathtub data. Communication in Statistics - Theory and Methods, 45(19), 5709-5729.

Teimouri, M., Hoseini, S. M., Nadarajah, S. (2013). Comparison of estimation methods for the Weibull distribution. Statistics, 47(1), 93-109.

\section{André Felipe Berdusco Menezes}

Departamento de Estatística . Universidade Estadual de Maringá, PR, Brasil Email: andrefelipemaringa@gmail.com Contribuição do autor

\section{Josmar Mazucheli,}

Departamento de Estatística . Universidade Estadual de Maringá, PR, Brasil

Email:jmazucheli@gmail.com

Contribuição do autor

\section{Kelly Vanessa Parede Barco}

Faculdade União de Campo Mourão PR, Brasil

Email: kekyvanessa@gmail.com

Contribuição do autor 